\title{
Publisher's Note: Linear magnetoelectricity at room temperature in perovskite superlattices by design [Phys. Rev. B 92, 184112 (2015)]
}

Saurabh Ghosh, Hena Das, and Craig J. Fennie

(Received 18 December 2015; published 30 December 2015)

DOI: 10.1103/PhysRevB.92.219906

PACS number(s): 75.85.+t, 71.15.Mb, 75.80.+q, 77.80.-e, 99.10.Fg

This paper was published online on 30 November 2015 with errors in Table III and some text on pages 8 and 9 . The four entries in the first column of Table III should have read as

$$
\begin{aligned}
& A_{x} G_{y} C_{z} \\
& F_{x} C_{y} G_{z} \\
& G_{x} A_{y} F_{z} \\
& C_{x} F_{y} A_{z}
\end{aligned}
$$

The last entry in the last column of Table III should read as " $\alpha_{x x}, \alpha_{y y}, \alpha_{z z}$." On page 8, right-hand column, lines seven and eight in the last paragraph should read as "reorientation from $G_{x} A_{y} F_{z}$ to $F_{x} G_{y} C_{z}$ around $480 \mathrm{~K}$. Moreover, $F_{x} G_{y} C_{z}$ is reported to induce ferroelectricity into the...." On page 9 , the first line of the left-hand paragraph should read as " $G_{x} A_{y} F_{z}$ to $F_{x} G_{y} C_{z}$ spin reorientation], which strengthens our...." The Table and text have been corrected as of 17 December 2015. The Table and text are incorrect in the printed version of the journal. 